\title{
Strain Distribution in Copper Tensile Specimens Prestrained in Rolling
}

\begin{abstract}
J.V. FERNANDES and M.F. VIEIRA
Sequences of orthogonal rolling-tension experiments were performed on polycrystalline copper sheets. The effect of strain path change on subsequent yield and flow behavior has been investigated. Optical microscopy and transmission electron microscopy (TEM) were used to clarify the physical mechanisms occurring during the second deformation. The observed increase in yield stress in reloading was related to the change of slip systems corresponding to the glide of dislocations with a Burgers vector, which had not been active during prestrain. The transient observed in the workhardening behavior after the path change corresponds to the appearance of disorganization in the dislocation microstructure. It was shown that no special feature of slip behavior inside the grains can be related to the nonhomogeneous surface deformation observed at the beginning of reloading. Also, the plastic instability of prestrained samples corresponding to the maximum load in tension does not seem to be directly controlled by the developed local substructure. The nonuniform deformation observed in reloading was studied using a simplified macroscopic two-zone model. It takes into account the presence of geometrical defects in the samples and considers the importance of the mechanical behavior. The macroscopic results, concerning the delay of starting deformation in some regions, are explained by the model, which allows formulation of an analytical condition necessary for deformation to spread through the length of the sample before necking takes place.
\end{abstract}

\section{INTRODUCTION}

WHEN the strain path is changed during the plastic deformation of metals, the flow behavior diverges from that typical of a monotonic deformation. A transient in the work hardening occurs after the change of strain path. Results from previous research concerning the mechanical behavior after reloading have been summarized as follows: $:^{[-4]}$

(1) a low initial flow stress (compared with the stress at the same equivalent strain in the monotonic path) is followed by a relatively high work-hardening rate; and (2) a high initial flow stress is followed by a lower workhardening rate.

The first case corresponds mainly to strain path changes associated with Bauschinger experiments (inversion of loading conditions). However, most behavior in complex strain paths concerns the second case, where the active slip systems change partially or totally after reloading.

For single-phase materials, the parameters expected to influence reloading behavior are the morphology of the grains, the crystallographic texture, and the dislocation microstructure. The importance of the morphology of the grains is not well studied; but it seems reasonable to support the idea that at moderate prestrain values (up to about 0.30 ), the change of the grain shape is not significant. As far as the evolution of the crystallographic texture is concerned, it is known that during deformation, it evolves slowly toward a "stable" orientation, which depends on the material

J.V. FERNANDES, Associate Professor, is with the Mechanics Department, Faculty of Science and Technology, Polo II of the University of Coimbra, 3030 Coimbra, Portugal. M.F. VIEIRA, Assistant Professor, is with the Metallurgical Department, Faculty of Engineering, University of Oporto, 4099 Porto Codex, Portugal.

Manuscript submitted March 28, 1996. and strain path. Generally, its influence in plastic behavior can be neglected at low strains. ${ }^{[5,6]}$

The effects of the existing microstructure on the behavior after reloading can be understood in terms of latent hardening and Bauschinger effect experiments. The first case corresponds to the activation of slip systems not activated during prestrain: the critical resolved shear stress of these systems is compared with that of systems operating during prestraining (the latent hardening ratio (LHR) is defined as the ratio of the critical resolved shear stresses in secondary and primary systems ${ }^{[7]}$ ). The LHR is lower for coplanar systems than for the intersecting systems. In the coplanar system, its value is quite independent of the prestrain amount and close to unity (at about 1.1 for copper ${ }^{[8]}$ ). For intersecting systems, the LHR value is clearly higher than unity, decreasing with increasing deformation (for copper, during stage II of deformation, values close to 1.3 have been found $\left.{ }^{[8]}\right)$. The latent hardening phenomena can be expressed in terms of dislocation-dislocation interactions. The change of slip system is associated with an increase in the forest dislocation density and a lack of mobile dislocations in the new active slip system. ${ }^{[9]}$ The Bauschinger experiments correspond to a change in strain path, such that the slip systems that have been active during the first path are still active in the second one, but in the opposite sense. The Bauschinger effect is associated with a low reloading stress. To explain this behavior it is supposed that the stress necessary to cause deformation has a directional component, which acts against deformation in the direction of the strain but aids deformation in the opposite direction. In support of this approach, one may consider that the dislocation structure is polarized: the cell walls present an excess of dislocations of one sign on each side of the wall. ${ }^{[10]}$ This makes the dissolution of the cell walls easy and promotes the annihilation of dislocations during reloading. In fact, experimental results show that the inversion of the loading 
direction makes the dislocation structures unstable; as a consequence, the increase in the density of mobile dislocations and the growth of the corresponding mean free path are sufficient to explain the relatively low level of stress after reloading in the opposite direction. ${ }^{[1,12,13]}$

When a strain path change occurs, the reloading behavior of the grains of the polycrystal is determined by latent hardening and Bauschinger effects. Previous work ${ }^{[14,15]}$ on copper has shown that for a sequence of two strain paths, behavior during the second loading, in tension, is highly dependent on the magnitude of the strain path change; this can be defined by the scalar parameter $\alpha$ that is equal to the cosine of the angle between the two vectors which represent the two strain path tensors. ${ }^{[16,17]}$ Particularly, after a critical prestrain value, the uniform strain during the second path in tension depends on the amplitude in the change of strain path, being independent of the nature of prestrain conditions, such as uniaxial tension, rolling, equibiaxial stretching, and shear. ${ }^{[14]}$

Several approaches have been used to understand the onset and development of macroscopic strain localization in copper. A phenomenological model has been proposed ${ }^{[14]}$ to predict the occurrence of early plastic instability in tension after prestrain. Other works ${ }^{[15,18]}$ have suggested that plastic instability occurring in tension after prestrain is only a mechanical consequence of the drop in the strain-hardening rate, which takes place at the beginning of reloading.

The present work examines the mechanical behavior and the microstructural evolution of copper sheets during orthogonal tension of cold-rolled samples. Dislocation patterns, slip lines, and morphology of surface strain distribution were analyzed. A study of active slip systems during reloading with respect to those previously active during prestrain was carried out to clarify the yield and flow behavior after path change. Eventual connections between slip behavior inside the grains and the surface strain distribution at the beginning of reloading are discussed. The development of necking is also analyzed as a consequence of the instability corresponding to maximum load.

\section{EXPERIMENTAL}

Oxygen-free copper (99.95 pct $\mathrm{Cu}$ ) sheets, 1-mm thick, as received under cold-rolled and annealed conditions, were used in this investigation. The mean grain size was $7 \mu \mathrm{m}$; the grains were equiaxed.

Sequences of two strain paths were performed at room temperature. The prestrain path was a unidirectional rolling. Square samples $\left(10^{4} \mathrm{~mm}^{2}\right)$ were deformed up to von Mises equivalent strains $\left(\varepsilon=(2 / \sqrt{3})\left(\varepsilon_{1}^{2}+\varepsilon_{2}^{2}+\varepsilon_{1} \varepsilon_{2}\right)^{1 / 2}\right.$, where $\varepsilon_{1}$ and $\varepsilon_{2}$ are the principal plastic strains in the sheet plane*)

\footnotetext{
*In rolling, de Von Mises equivalent strain $\varepsilon=(2 / \sqrt{3}) \varepsilon_{3} \approx 1.155 \varepsilon_{3}, \varepsilon_{3}$ being the thickness strain. In tension, the equivalent strain $\varepsilon=\varepsilon_{1}, \varepsilon_{1}$ being the strain in the tensile direction.

between 0 and 0.26 in rolling equipment with a $72.5-\mathrm{mm}$ roll diameter and using a roll speed of 20 rotations per minute in such a way that a strain of 0.01 needs about three passes. The second path was in uniaxial tension and was performed on samples of gage length and width of 60 and $10 \mathrm{~mm}$, respectively, cut from the middle of the large prestrained specimen. The tensile axis was normal to the rolling
}

direction. The tensile tests were performed using an Instron machine equipped with a $50-\mathrm{mm}$ strain gage. The initial strain rate was $\varepsilon=7 \times 10^{-4} \mathrm{~s}^{-1}$ and the strain amounts were deduced from extensometer data. A microcomputer was interfaced with the tensile testing machine, and signals from load and extension were stored and analyzed digitally.

The thickness and width of the tensile samples were carefully measured using a traveling microscope, which had an accuracy of $1 \mu \mathrm{m}$, to check the presence of dimensional fluctuations. A precise measure of the thickness is important because it can give information about the homogeneity of the rolling process. Samples with several strain amounts in rolling (namely, $0,0.10,0.20$, and 0.30 ) were mounted and polished in order to have a good definition of the entire section. Before mounting, the samples were fixed with clips in such a way that the normal direction was parallel to the observation plane. The rolling process was very homogeneous, i.e., no significant fluctuations in thickness were measured even for strains higher than that one used in this work: the amplitude of the fluctuation observed in thickness was always of the order of $20 \mu \mathrm{m}$. The variation in width, coming from the machining, is such that the test specimen shows differences close to $100 \mu \mathrm{m}$ (these values are allowed by standardization). So, the variation in area $\Delta A_{0}$ is always about $0.30 \mathrm{~mm}^{2}$, for an initial area of $A_{0}=10 \mathrm{~mm}^{2}$ $\left(\Delta A_{0} / A_{0}=0.03\right)$.

To characterize strain distribution during the second path in tension, some of the samples were carefully polished after prestraining. The specimens were electropolished by entire immersion in a solution containing $250 \mathrm{~mL}$ orthophosphoric acid, $250 \mathrm{~mL}$ ethanol, $500 \mathrm{~mL}$ distilled water, and $3 \mathrm{~g}$ urea and using a current density of $80 \mathrm{~A} / \mathrm{dm}^{2}$. Series of photographs were taken during the tensile tests of these samples.

The microstructure of the deformed sample was observed through light microscopy and transmission electron microscopy (TEM) $(100 \mathrm{kV})$. The observation plane was the sheet plane. Sample preparation has been described elsewhere. ${ }^{[15]}$

\section{RESULTS}

\section{A. Mechanical Behavior}

Examples of nominal stress $(\Sigma)$-nominal strain $(e)$ curves obtained in tension are shown in Figure 1. After prestrain equal to or greater than 0.06 , the curves show a clear transient region with concavity upward; the magnitude of this transient increases with increased prestrain. Samples with prestrain values of 0.08 and 0.10 exhibited two load peaks: a sharp peak near yielding and a broad peak at higher strain. After the sharp peak, the slope of the corresponding true stress-true strain curves (Figure 2) are greater than zero, which means that the deformation is not of the Luders band type. For prestrain values of 0.12 and higher, only one maximum of load is observed just after the yielding.

The true stress-true strain curves along the reference direction (the transverse direction of the sheet) have been drawn for all the prestrain amounts. In Figure 2, examples of these curves for prestrain values of $0.04,0.08,0.12,0.16$, and 0.20 are presented. In the same figure, the reference curve (without prestrain) is plotted. After path change, the yield stress (which may be defined as the back extrapolated 


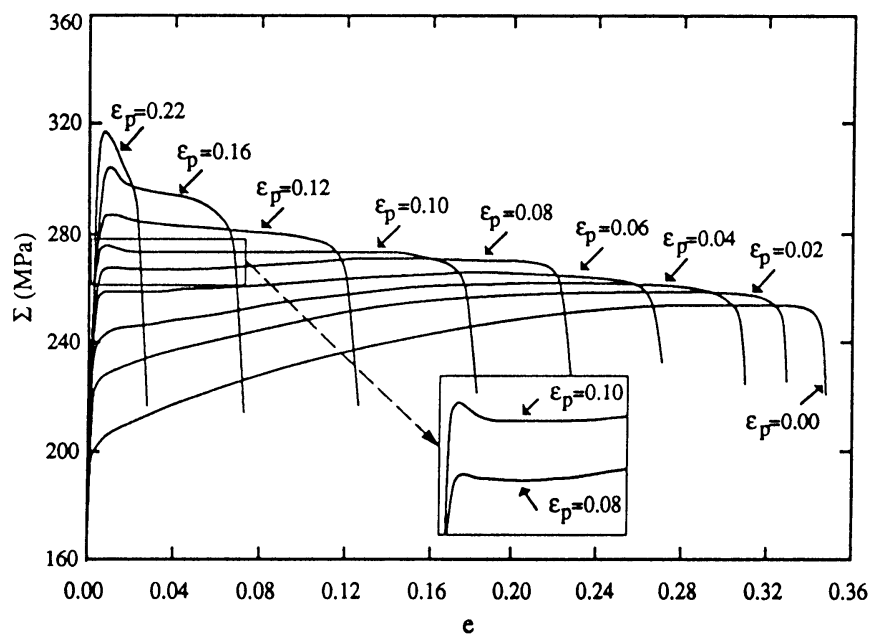

Fig. 1-Examples of nominal stress $(\Sigma)$-nominal strain $(e)$ curves in tension of samples prestrained in rolling. The curve without prestrain $\left(\varepsilon_{p}\right.$ $=0)$ is also shown.

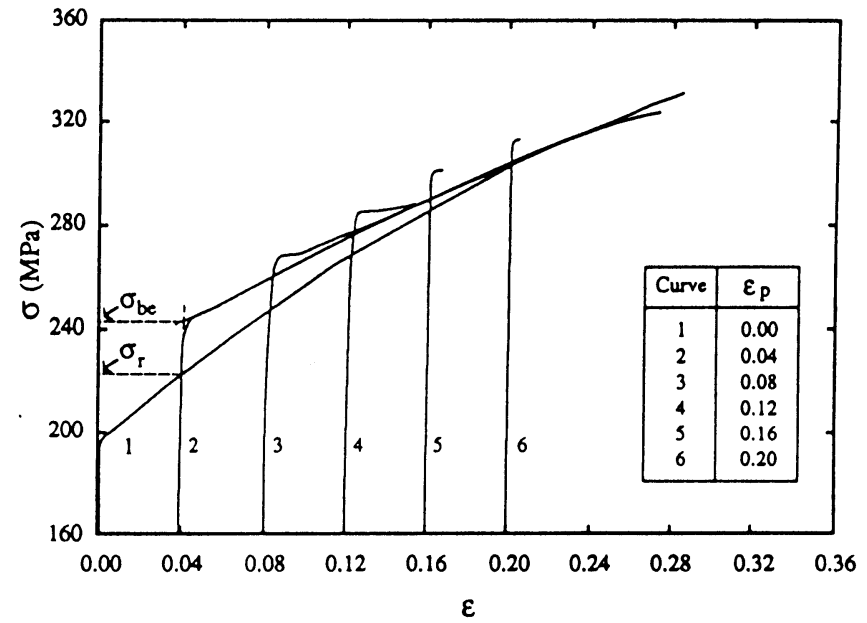

Fig. 2-Examples of true stress $(\sigma)$-true strain $(\varepsilon)$ curves in tension of samples prestrained in rolling. The curve without prestrain $\left(\varepsilon_{p}=0\right)$ is also shown.

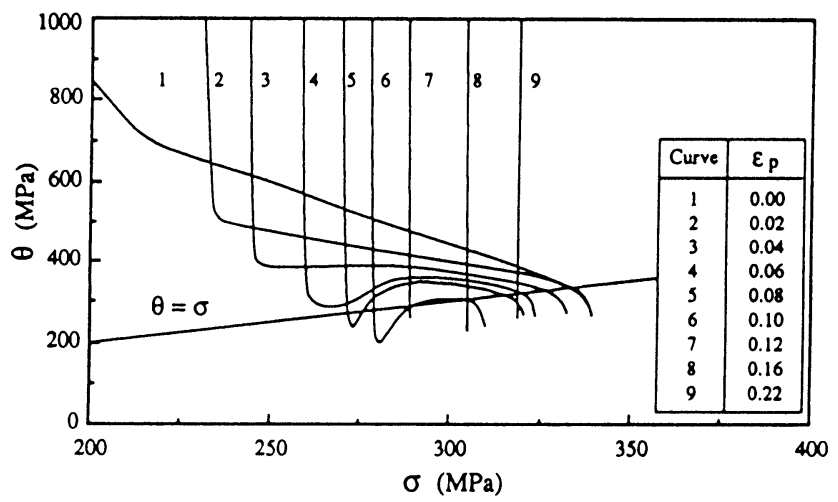

Fig. 3-Evolution of the work-hardening rate $\theta=d \sigma / d \varepsilon$ with the true tensile stress $\sigma$

stress $\left.\sigma_{b e}^{[14,15,17]}\right)$ is higher than the stress reached on the reference curve $\left(\sigma_{r}\right)$ at a strain which is equal to the prestrain amount. The reloading yield stress is followed by a transient region with a low strain-hardening rate. This effect
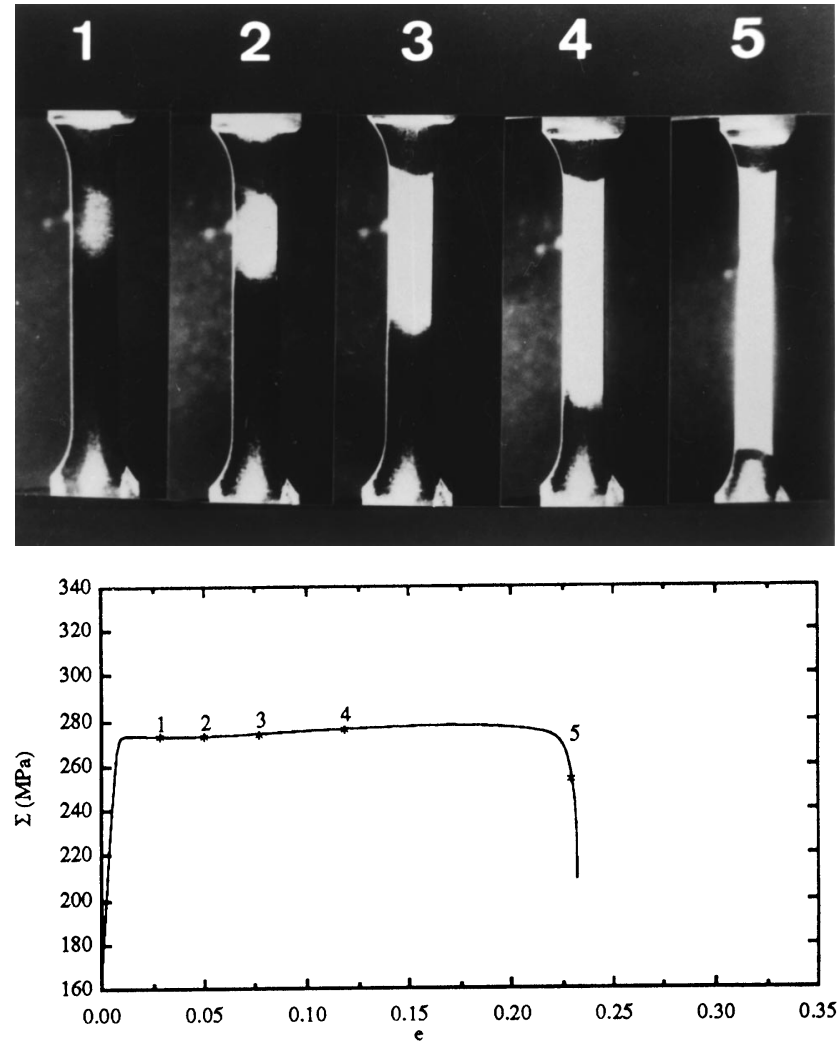

Fig. 4-Evolution of the deformed region in tension after prestrain in rolling up to $\varepsilon_{p}=0.08$.

is more clearly seen if we plot the evolution of the workhardening rate $\theta$ with the tensile stress $\sigma$ (in Figure 3, some examples are shown). At a given stress, the work-hardening rate is lower after reloading than for a monotonic test; this effect is greater for large prestrain values. Also, as long as prestrain increases, the curves become more horizontal, i.e., the work-hardening rate remains almost constant in a large range of deformation. This behavior is a consequence of the tendency that shows the stress-strain curves of prestrained samples to approach asymptotically the monotonic one.

\section{B. Macroscopic Strain Distribution}

Photographs were taken during the tensile tests of samples specially prepared by polishing to permit the evolution of the deformed region to be examined. This region appears bright in the photograph because its superficial relief reflects the light in a different way from the nondeformed region. In the samples without prestrain, the bright region covers the entire specimen and can be observed after a deformation of about 0.03 . For prestrained samples, the results are summarized as follows.

(1) For low prestrain values ( 0.04 and below), a homogeneous deformation is observed in the sample. Sometimes, a small gap of deformation is necessary before the sample is seen to deform along the entire gage length.

(2) When the prestrain value is in the range 0.06 to 0.10 , one region where deformation begins is clearly observed (or at least where deformation is seen macroscopically to begin), as is shown in Figure 4. This region spreads with deformation, but rupture has been 
seen to arise before the entire sample has deformed, in the case of a prestrain value of 0.10 .

(3) For prestrain values where only one peak load appears immediately after yielding (highest prestrain values), the observed localized region has a fairly defined profile from the start of deformation (the greater the prestrain) and spreads just a little before rupture (Figure 5).

\section{X-ray Texture Measurements and Anisotropy}

$\mathrm{X}$-ray analysis ${ }^{[19]}$ was performed on samples without deformation and deformed up to 0.20 in rolling. For the three samples, $\{111\},\{200\},\{220\}$, and $\{311\}$ incomplete pole figures were measured. To go further in analyses, the quantitative three-dimensional orientation distribution functions (ODFs) have been determined for each sample. Examples of relevant sections of ODF without deformation and deformed up to 0.20 in rolling are presented in Figure 6. From the experimental ODFs, it was possible to define the main texture components. The results are summarized in Figure 7 , which shows the distribution of the rolling direction in $\{110\},\{112\}$, and $\{123\}$ planes, respectively, parallel to the sheet plane.

The initial texture of the sheet is quite smooth. Nevertheless, it is possible to distinguish several texture components such as $\{110\}\langle 1 \overline{1} 0\rangle, \quad\{110\}\langle 1 \overline{1} 1\rangle, \quad\{110\}\langle 5 \overline{5} 8\rangle$, $\{112\}\langle 1 \overline{1} 0\rangle$, and $\{123\}\langle 3 \overline{3} 1\rangle$. During prestrain in rolling, up to 0.20 , the texture almost does not evolve. These results agree with the value of the $R$ factor determined experimentally, which is close to unity $(R=0.98)$.

\section{Structural Observations}

\section{Transmission electron microscopy}

During deformation, dislocations tend to arrange themselves to form walls defining cells in which there is a lower dislocation density. The following remarks refer to observations of several thin foils (more than five) for each deformation state referred to; this corresponds to about 30 grains for each case.

The TEM observations, after deformation in rolling, have shown that the dislocation microstructure in the sheet plane consists of a loose cell structure (Figure 8) up to strain values of 0.26 , independent of crystallographic orientation.

In tension without prestrain, the dislocation cell structure is generally better defined: the dislocation wall thicknesses and the dislocation density in the cell interior are smaller for the same strain value. Besides, two intersecting families of parallel dislocation walls are visible in some grains with particular orientation.

At the beginning of reloading in tension after prestrain in rolling, the dislocation microstructure was still more disorganized than that in rolling (Figure 9). When it was possible to attain large deformation in the subsequent tension, two intersecting families of parallel straight walls were observed a few times in the macroscopic strain localization region (Figure 10), as in tension without prestrain.

\section{Optical microscopy}

During the second test in tension, the slip lines were observed through an optical microscope by differential interference contrast. In order to follow the evolution of slip line pattern during deformation, observations were per-
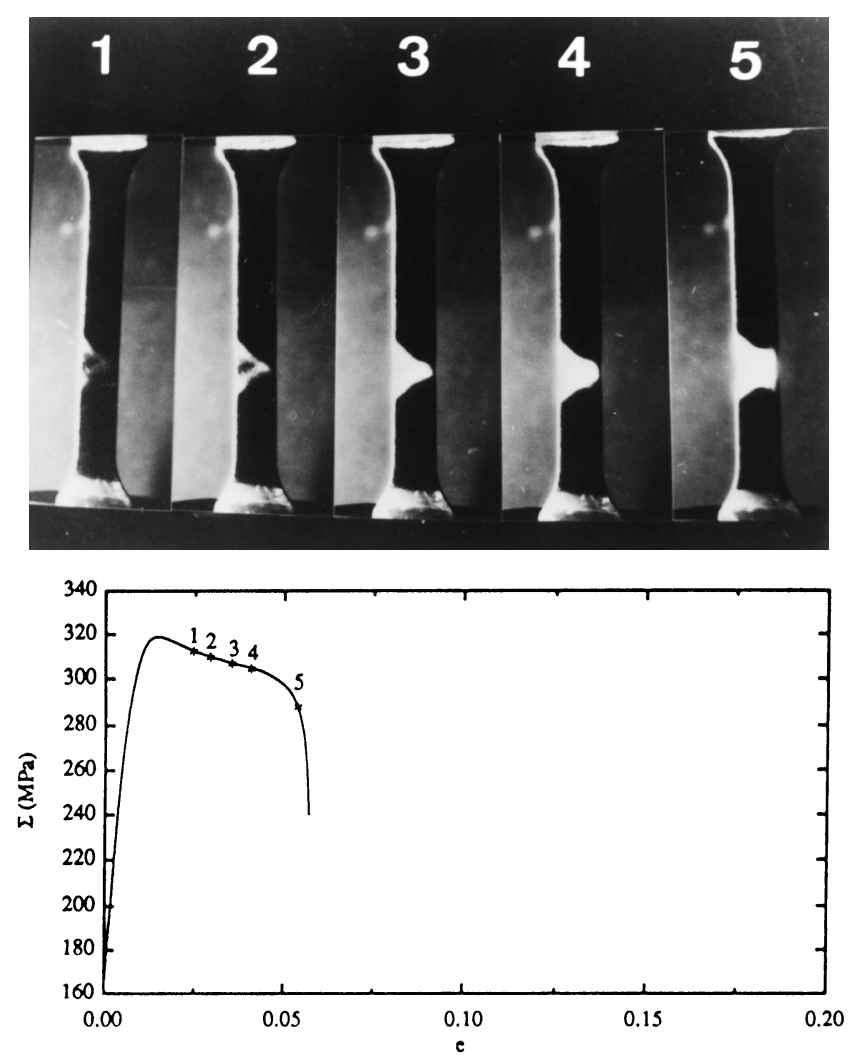

Fig. 5-Evolution of the deformed region in tension after prestrain in rolling up to $\varepsilon_{p}=0.22$.

formed after several strain values; also, several prestrain values (including zero) were used. The results are summarized as follows.

(1) One slip system (set of parallel slip lines in a grain) was observed in most of the grains (Figure 11). Some grains showed other families of slip lines in restricted areas.

(2) The orientation distribution of the slip line system directions was determined. A statistical analysis was performed taking into consideration all the slip line systems visible in photographed regions (for example, Figure 10). Almost all of the slip line system directions (about 99 pct in a total of 1500 measurements) are inclined to the tensile axes of an angle between 30 and $90 \mathrm{deg}$ ( 70 pct between 40 and $70 \mathrm{deg}$ ).

(3) The two preceding results do not depend on the prestrain value, the strain amount in tension, or the observed region.

(4) Regions of localized deformation at the grain level were not observed. The slip lines do not cross the grain boundaries; these did not show offsets or steps, even in the necking region of the highest prestrained samples.

Also, optical observations in the cross section of the sample after rolling did not reveal particular deformation markings or imperfections in strain distribution.

\section{DISCUSSION}

The results discussed in Section III show the effect of prestrain value in unidirectional rolling on the subsequent 

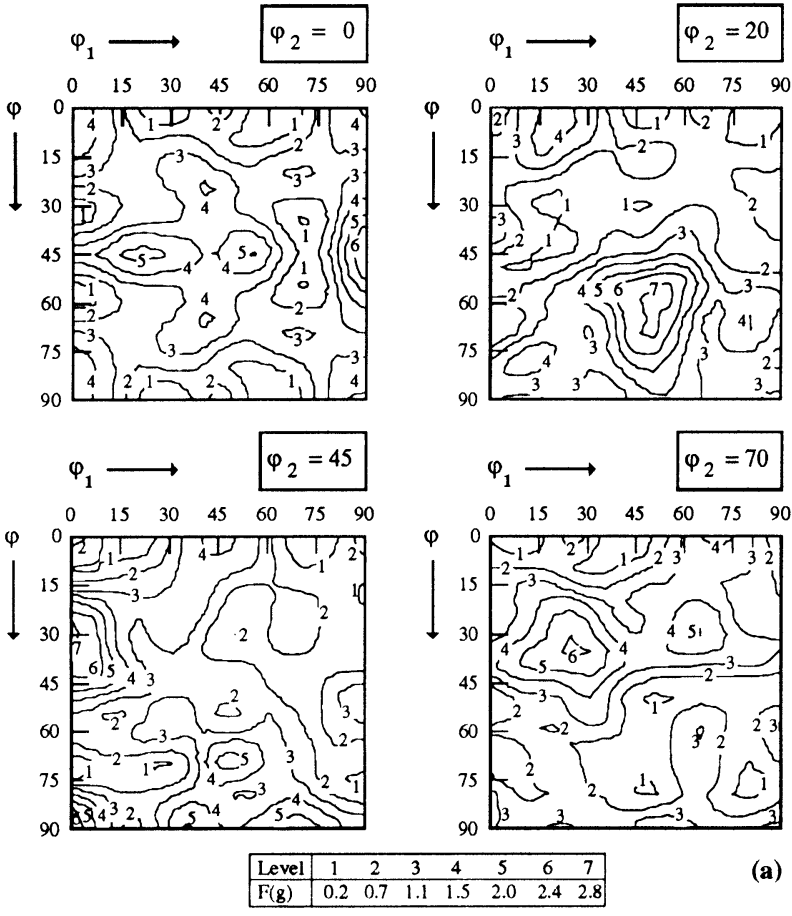
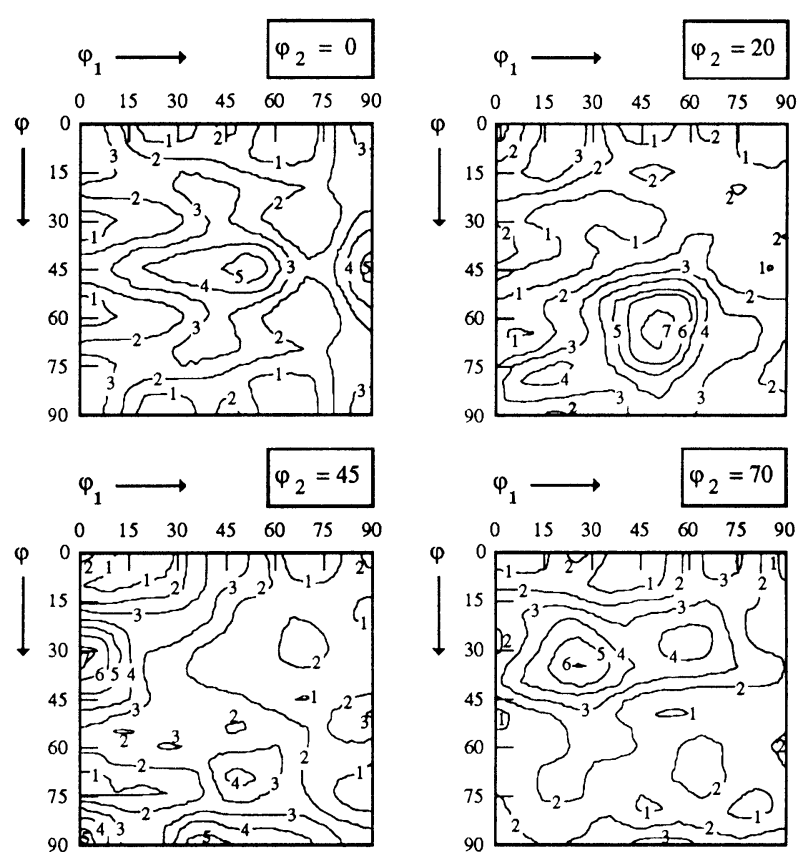

90
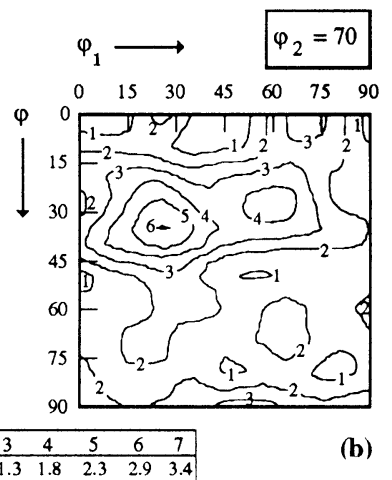

Fig. 6-ODF sections of the copper sheet for $\varphi_{2}=0,20,45$, and 70 deg: $(a)$ without deformation and $(b)$ deformed up to 0.22 in rolling.

behavior of copper sheets during tensile deformation along an orthogonal direction. It is clear that the amount of prestrain influences the strain value at which mechanical instability appears, corresponding to the maximum load: this instability arises just after yielding for 0.12 and higher prestrain values. For slightly lower values $(0.08$ and 0.10$)$, two load peaks were observed: a sharp peak near yielding and a broad peak at higher strain. Another remarkable feature is that for prestrain values equal to or higher than 0.06 , the strain distribution is not homogeneous in the tensile sample, even before the maximum load has been attained. Light (slip lines) and electron transmission (dislocation configurations) microscopes were performed in order to discuss microstructural explanations of these facts. The macroscopic aspects of the described behavior will be analyzed also in Section B.

\section{A. Microstructural Aspects}

\section{Dislocation structure}

The dislocation substructure that develops during plastic deformation of polycrystalline copper has been extensively studied (in References 18, 20, and 21, for example). After uniaxial tension tests, only one set of long parallel dislocation walls are generated for samples with the larger grain size (about $250 \mu \mathrm{m}$ ), and a closed cell structure (rectangular or equiaxed cells) appears for those with small grain size (less than $50-\mu \mathrm{m}$ diameter). ${ }^{[20]}$ These observations indicate that for large grain sizes, the majority of the grains behave as a single crystal and deform mostly in single slip, i.e., one slip system is clearly more active than the others. For small grain sizes, homogeneous multislip conditions prevail in the entire grain, which lead to a closed cell structure. This is the result of internal stress due to the accommodation process between adjacent grains. After rolling, the dislocation substructure consists of equiaxed cells for both 20and $250-\mu \mathrm{m}$ grain sizes. This behavior was assumed to be the result of strongly constrained deformation imposed by the strain path in rolling itself. So, the developed dislocation structure is a result of coupling between the grain size and the loading conditions. In rolling, independent of the grain size (up to $250 \mu \mathrm{m}$ ), and in tension for small grain sizes (less than $50 \mu \mathrm{m}$ ), the intergranular interactions, raising the number of active slip systems, approach the Taylor condition of homogeneous deformation. ${ }^{[20]}$

During complex strain paths, two main cases of microstructural modifications have been observed after path change, depending on the material, grain size, and type of path change. In some cases, microbands appear inside the grains at the first stages of deformation after yielding; $;{ }^{[18,21-}$ ${ }^{23}$ in other cases, the dislocation microstructure evolves gradually, by dissolution of the prestrain structure toward that which is typical of the current path, and no trace of microscopic localized deformation is noted. ${ }^{[13,15,18,24]}$ Recent work ${ }^{[18,21]}$ suggests that a necessary condition for microband development, during the reloading of a prestrained sample, is the occurrence of intense glide on one slip system that was inactive during the prestrain (as in tension after orthogonal rolling). This allows further understanding of why microbands have been observed, after path change, in steel with small grain size (at about $30 \mu \mathrm{m})^{[23,25]}$ for which the pencil glide favors the accommodation process in the grains, and thus, intense activity in one slip system can occur. $^{[26]}$ Also, in copper with large grain size $250 \mu \mathrm{m}$ (where grains behave, for the most part, as single crystals: ${ }^{[20]}$ only one slip system is mainly active), microbands have been observed at the beginning of the second path in the following sequences: rolling followed by orthogonal tension, ${ }^{[18]}$ tension-tension with 54 deg between the two tensile axes, and tension-shear with the shear direction normal to the tensile axis. ${ }^{[21]}$

In the present case, TEM observations have shown mainly the presence of closed equiaxed cell structure, what- 

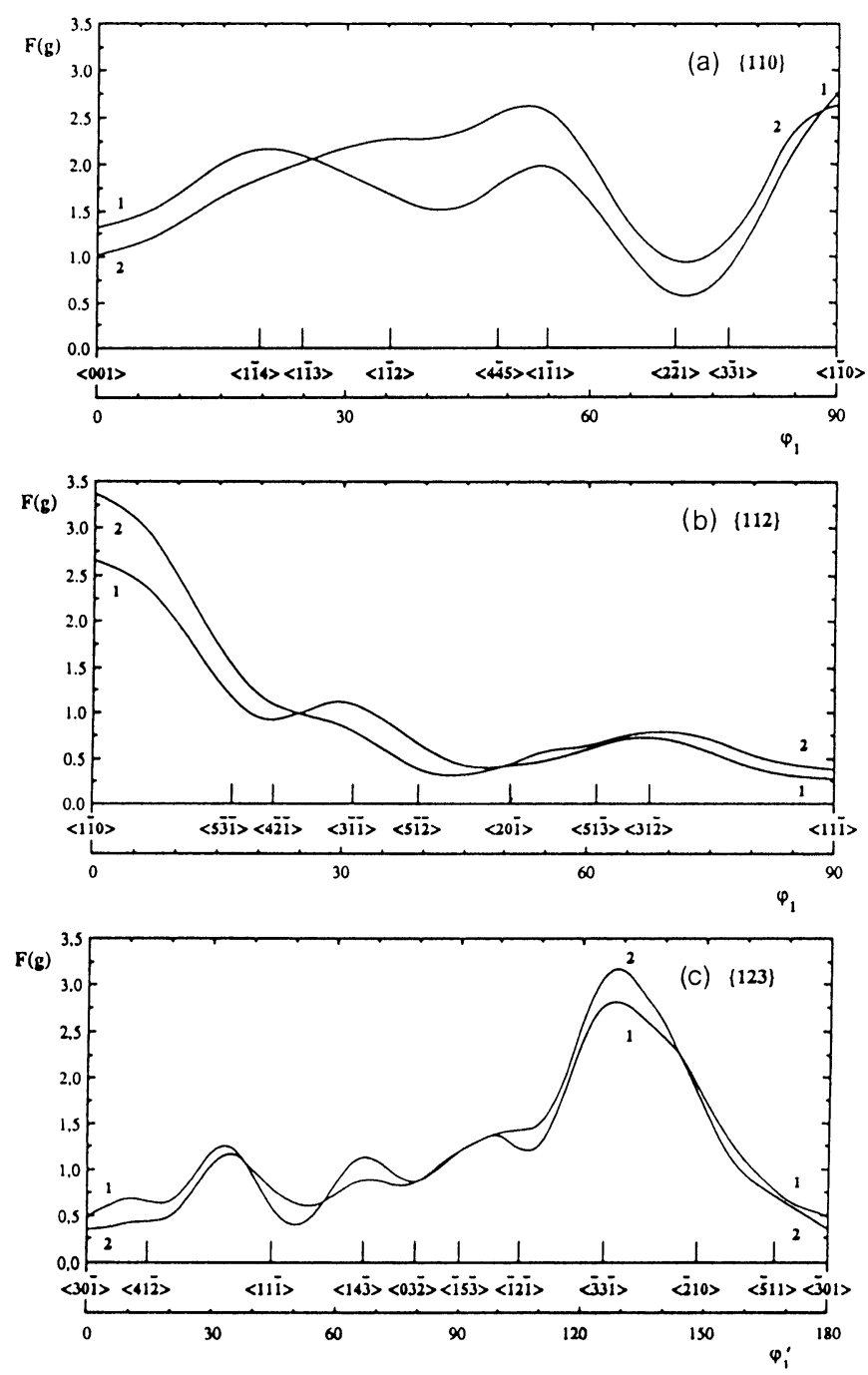

Fig. 7-Variation of the ODF $F(g)$ when the rolling direction remains in the $(a)\{110\},(b)\{112\}$, and (c) $\{123\}$ planes parallel to the sheet plane: curve 1 -without deformation; and curve 2 -deformed up to 0.22 in rolling.

ever the strain path in rolling or in tension with or without prestrain. This indicates a homogeneous intragranular deformation. In fact, for so small a grain size (about $7 \mu \mathrm{m}$ ), the strain accommodation process is complex: the grains are strongly influenced by their surroundings and three or more non-coplanar systems have equivalent activities. This agrees with the idea that the behavior of fine-grain material agrees with the Taylor approach to multiple slip. ${ }^{[27]}$

Moreover, the plastic strain inside the grains is stable, i.e., no localized shear associated with microbands or any other instabilities have been observed, even after prestrain, contrary to that observed for large grain size, as has been reported previously for copper. Also, no shear offsets were noted in the grain boundary regions: the compatibility conditions near the grain boundaries can be satisfied by the operation of supplemental systems in this region. ${ }^{[28,29]}$

\section{Surface structure}

The surface relief structure (Figure 11) revealed closed active parallel slip planes. The orientation of the slip lines in the grains does not depend on the observed region. Most of them are inclined to the tensile axes at an angle between

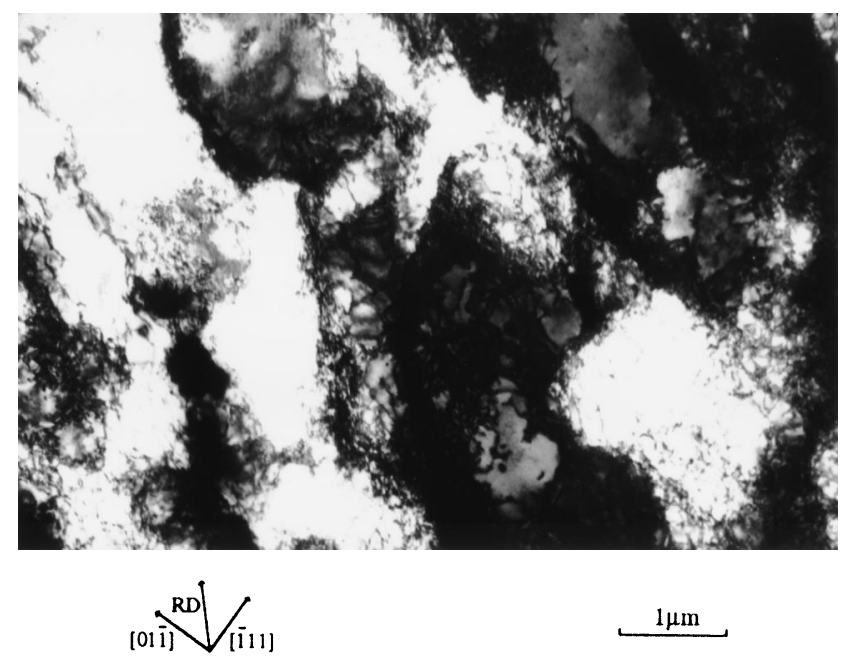

Fig. 8-TEM observation of closed cell structure after a strain amount in rolling equal to 0.26 in a grain with $\langle 112\rangle$ axis normal to the sheet plane (RD-rolling direction).

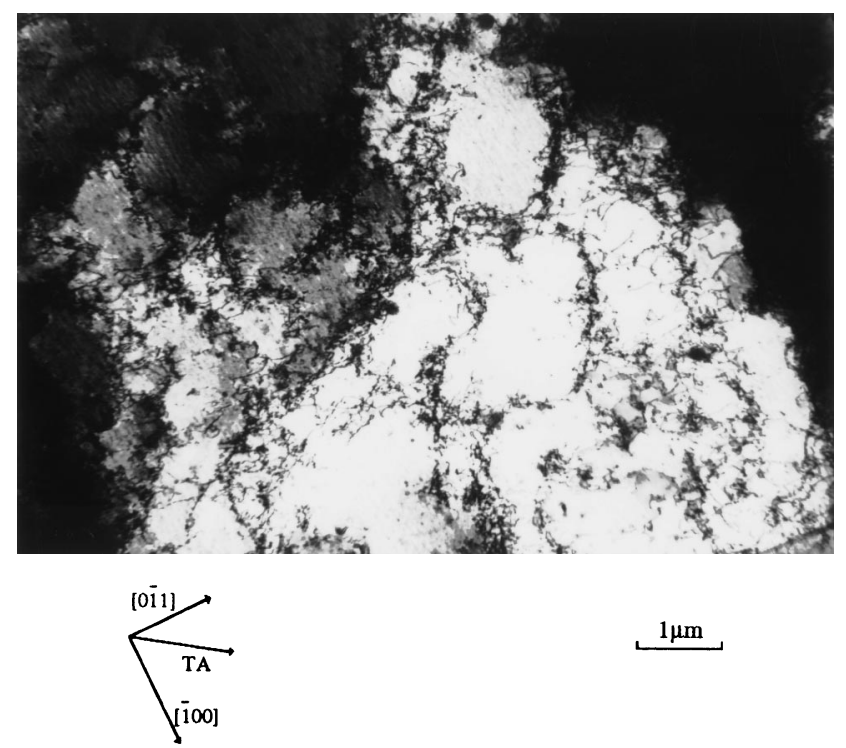

Fig. 9-TEM observation of unorganized dislocation structure, developed during the second path in tension (strain approximately equal to 0.02 ) in a grain with $\langle 110\rangle$ axis normal to the sheet plane. The prestrain value in rolling is equal to 0.16 . (TA-tensile axis, normal to the rolling direction.)

30 and $90 \mathrm{deg}$, as has already been mentioned in the cases of monotonic tension and tension-tension experiments on copper sheets with $20-\mu \mathrm{m}$ grain size. ${ }^{[15]}$ In particular, this kind of slip line distribution is noted inside the necking region, which confirms that there is no special configuration in the region of strain localization, contrary to that observed in steel submitted to the same strain path. ${ }^{[25]}$ For steel, the slip lines (referred to as microshear bands because they cross more than one grain) are parallel to the macroscopic band of localized deformation.

The comparison between surface (optical) and bulk (TEM) observations described previously indicates that, apparently, the number of active slip systems in surface grains is less than in interior grains. Most of the surface grains show just one set of parallel slip lines, while the closed dislocation cell structure observed in the bulk grains indicates the activity of, at least, two non-coplanar slip systems. 

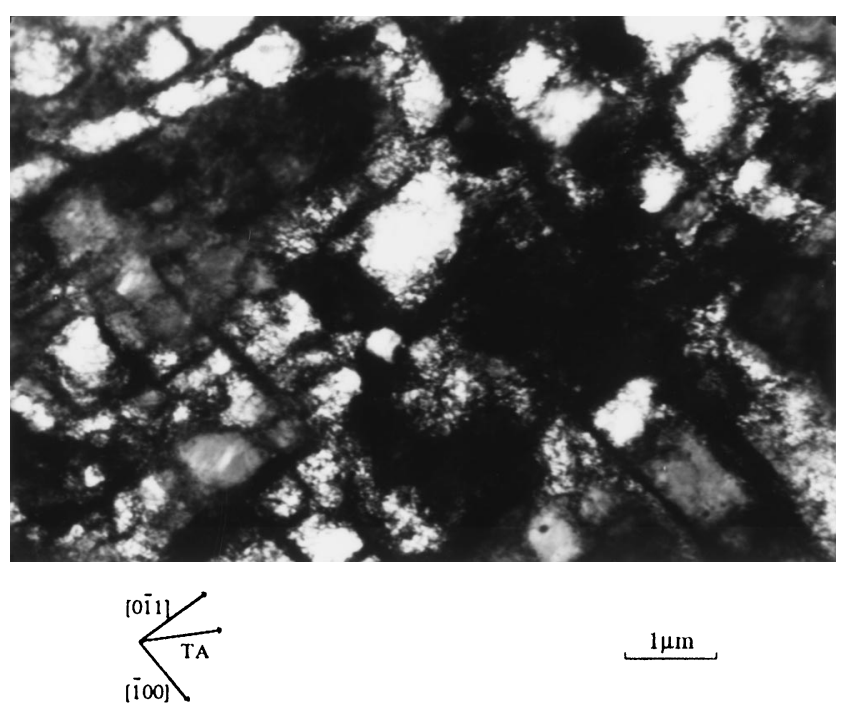

Fig. 10-TEM observation of closed parallelogram cells developed near the rupture line during the second deformation in a grain with $\langle 110\rangle$ axis normal to the sheet plane. The prestrain value in rolling is equal to 0.16 (TA-tensile axis, normal to the rolling direction.)

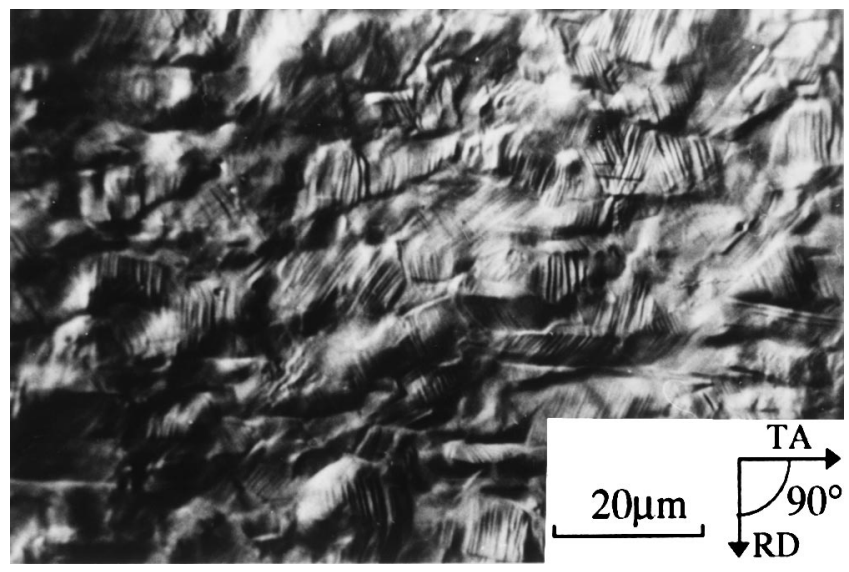

Fig. 11-Optical observation of slip lines, developed during the second deformation in the macroscopic localized region, shown in Fig. 5 (after rupture). The prestrain value is equal to 0.22 .

Two main reasons can explain this fact: the grains at the surface are less constrained than the others; ${ }^{[27,29]}$ moreover, surface observation methods cannot reveal all the active slip systems. In fact, the slip activity needed to make the slip lines visible by optical microscopy depends on the relative orientation between slip plane and surface plane observation (in some grains, the slip lines are not visible before there is a strain value equal to or slightly greater than 0.10 ). Besides, an observed slip line system may correspond to two independent coplanar slip systems. However, from a qualitative point of view, the surface relief structure can be used as a complement of the dislocation structure in the bulk. In particular, in a previous work, ${ }^{[15]}$ the surface relief observations were used adequately in order to follow the change of active slip systems during reloading in sequential tension tests.

Macroscopic shear bands can develop during rolling. ${ }^{30,31]}$ In copper, these shear bands have been observed only for rolling deformations higher than 0.5 , which is a value much superior to that associated with the instability processes
Table I. Sets of Active Slip Systems According to the Taylor Model in Rolling and Tension (Normal to Rolling) for Five Grain Orientations Corresponding to the Main Texture Components

\begin{tabular}{|c|c|c|c|}
\hline Case & Texture Component & Rolling & Tension \\
\hline 1 & $(123)<3 \overline{3} 1>$ & $\overline{\mathrm{A} 3} \mathrm{C} 3$ & $\frac{\mathrm{A} 2}{\mathrm{~A} 6} \frac{\overline{\mathrm{B}} 4}{\mathrm{~B} 2} \mathrm{D} 6$ \\
\hline 2 & $(112)<1 \overline{1} 0>$ & $\overline{\mathrm{A} 3} \overline{\mathrm{D} 1}$ & $\frac{\mathrm{A} 2}{\mathrm{~A} 6} \frac{\overline{\mathrm{B}} 4}{\mathrm{~B} 2} \mathrm{D} 6$ \\
\hline 3 & $(110)<1 \overline{1} 0>$ & $\begin{array}{l}\overline{\mathrm{A} 3} \mathrm{~B} 4 \\
\mathrm{C} 3 \overline{\mathrm{D} 4} \\
\overline{\mathrm{A} 2} \mathrm{C} 1 \\
\mathrm{~B} 2 \overline{\mathrm{D} 1}\end{array}$ & $\begin{array}{llll}\mathrm{A} 2 & \mathrm{~B} 4 & \mathrm{C} 3 & \mathrm{D} 1 \\
\mathrm{~B} 4 & \mathrm{C} 1 & \mathrm{D} 1 & \mathrm{D} 4 \\
\mathrm{~A} 2 & \mathrm{~A} 3 & \mathrm{~B} 2 & \mathrm{C} 3 \\
\mathrm{~A} 3 & \mathrm{~B} 2 & \mathrm{C} 1 & \mathrm{D} 4 \\
\mathrm{~A} 2 & \mathrm{~B} 2 & \mathrm{~B} 4 & \mathrm{D} 4 \\
\mathrm{~A} 3 & \mathrm{~B} 2 & \mathrm{C} 3 & \mathrm{C} 5 \\
\mathrm{~A} 3 & \mathrm{C} 1 & \mathrm{C} 3 & \mathrm{D} 1 \\
\end{array}$ \\
\hline 4 & $(110)<1 \overline{1} 1>$ & $\begin{array}{c}\mathrm{B} 2 \mathrm{C} 3 \\
\mathrm{~A} 2 \mathrm{~B} 2 \mathrm{C} 3 \\
\mathrm{~A} 3 \mathrm{~B} 2 \mathrm{C} 3 \\
\end{array}$ & B4 B5 C1 C5 \\
\hline 5 & $(110)<5 \overline{5} 8>$ & B2 C3 & $\begin{array}{l}\text { B4 C5 } \overline{\overline{D 1}} \\
\text { B5 } 1 \text { C1 } \\
\end{array}$ \\
\hline
\end{tabular}

treated in this work (between 0.06 and 0.18). This is in concordance with our analyses of surface observations which indicate that no shear bands have been observed for the rolling deformation levels that we use in this work.

From the preceding analysis, it can be concluded that the appearance of instability corresponding to the maximum load cannot be associated with any specific observation of localization at the grain level, contrary to that reported for mild steel during the same loading sequence. ${ }^{[25]}$

\section{B. Macroscopic Aspects}

\section{Mechanical behavior}

The observed increase in yield stress after path change can be related to the physical mechanisms that occur during subsequent deformation. The evolution of the crystallographic texture during prestrain in rolling does not explain this hardening: there is almost no evolution of the texture during prestrain in rolling, up to 0.20 (Figure 7). Thus, the latent hardening effect plays an important role in this behavior, which can be analyzed by investigating the nature of the active slip systems during the two successive strain paths. ${ }^{\left[{ }^{15}, 18\right]}$ Assuming that the intragranular behavior follows the Taylor model, ${ }^{[20]}$ in agreement with TEM observations, the sets of possible active slip systems during the two successive paths have been estimated for different grain orientations. Results for five cases, corresponding to the main components of the texture in the sheets, are given in Table I. In this table, the possible active slip systems are listed (labeled using the Schmid and Boas notation) when they account for more than 20 pct of the total shear, in at least one set of Taylor solutions; this explains the fact that in some cases, less than five slip systems are listed. The change in the active slip systems, which occurs after reloading, is thus easily seen: the most active slip systems during prestrain and during subsequent tension are different; in most cases, the occurrence of a new slip system corresponds to the slip of dislocations with a Burgers vector, which has not been activated during prestrain. Only in the case of the texture component $\{110\}\langle 1 \overline{1} 0\rangle$ is one slip 


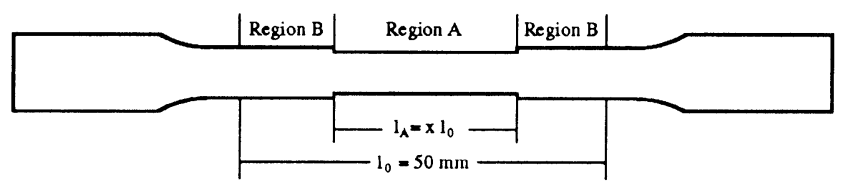

Fig. 12-Tensile sample considered in the model, showing regions A and $\mathrm{B}$ with different cross-sectional areas.

system active in both paths, although three new slip systems are active after strain path change. The change of slip systems corresponds to the slip of dislocations with a Burgers vector, which has not been activated during the prestrain. The same conclusions have been obtained for other grain orientations, less represented in the polycrystal. Due to the latent hardening effect, i.e., the relatively higher value of the resolved shear stress in the slip systems not active during prestrain, the yield stress increases after path change. ${ }^{[15,18]}$

After yielding, a relatively low work-hardening rate $(\theta$ $=d \sigma / d \varepsilon$, where $\sigma$ and $\varepsilon$ are the true stress and strain, respectively) is observed during the early stage of reloading, as shown in Figure 3. This is a result of two phenomena: the rapid increase of mobile dislocation density on the new active slip systems, most of them with a new Burgers vector; and the decrease in forest dislocation density due to a strong dynamic recovery of the previous structure. In fact, in their early work, Washburn and Murty ${ }^{[32]}$ showed that when the deformation conditions change, the existing dislocation structure becomes unstable: the passage of groups of dislocations into dislocation tangles tends to change their organization and promotes annihilation of dislocations. They pointed out that during tension deformation of copper single crystals oriented for single slip, after prestrain by multiple slip, the previous dislocation structure is gradually replaced by a structure typical of the new path, i.e., with greater stability under the new loading conditions. This causes the appearance of a transient period in work-hardening behavior, after which the strain-hardening rate takes values close to those observed in monotonic tension at the same stress (Figure 3). In the transient period, the strainhardening rate can overtake a minimum, if the prestrain value is high enough. Moreover, the work-hardening behavior obtained after path change is expressed, for prestrain values equal to 0.08 and 0.10 , by the presence of two load peaks (Figure 1), as observed in shear experiments after orthogonal strain path change. ${ }^{[33]}$ This is behavior not commonly observed in tension: generally, once instability corresponding to the maximum load (Considere point, for which the condition $\theta=\sigma$ is verified) is attained, the process of deformation localization, which accompanies the drop in load, initiates and continues without interruption up to rupture. In the present case, when two load peaks are observed, the minimum strain-hardening rate is attained at the start of deformation, when it drops below the straight line $\theta=\sigma$; after this, a strong recuperation in strain-hardening behavior occurs and the hardening curve jumps above the line $\theta=\sigma$ (Figure 3 ).

\section{Strain distribution}

In tension, the phenomenon of flow localization is recognized as a precursor to failure. During plastic deformation of metals, the flow localization has been associated either with local (at the grain level) or macroscopic plastic instabilities. During complex strain paths, an early flow localization is often observed (in References 2, 14, 15, 17, and 24, for example). The present results agree with this. From the preceding discussion, it was concluded that the plastic instability in tension of prestrained samples seemed not to be directly controlled by the local substructure changes. In this context, it is useful to emphasize that there is a correlation between the hardening behavior and the strain distribution along the subsequent path.

The results concerning macroscopic strain evolution show that before the Considere point is attained, the observed deformation after prestrain can occur in a limited region in the sample which spreads out before the final localization of deformation takes place. This is almost not observed after prestrain values equal to or less than 0.04 , which may indicate that the relatively high strain-hardening rate, observed at the beginning of the second path after low prestrain values, favors uniform deformation. For higher prestrain values, the nonuniform deformation, observed at the beginning of the second path in tension, is surely connected with the correspondent work-hardening behavior and with fluctuations in the area of the cross section of the sample, due to rolling and subsequent machining. In fact, the presence of geometrical defects induces a nonuniform macroscopic plastic deformation process in metals. The way in which the strain distributes depends on the size of the geometrical defect and on the work-hardening behavior, as discussed subsequently.

A simplified model to predict the strain distribution is to consider that the sample presents two regions, each one with a constant cross-sectional area, as shown in Figure 12. Besides, for low strain increments, the work-hardening behavior after path change can be considered close to linearity:

$$
\sigma=\sigma_{b e}+\theta \varepsilon
$$

Under these conditions, the applied force $F$ is given by the equation

$$
F=F^{\mathrm{A}}=\left(\sigma_{b e}+\theta \varepsilon^{\mathrm{A}}\right) A^{\mathrm{A}}=\left(\sigma_{b e}+\theta \varepsilon^{\mathrm{A}}\right) \frac{A_{0}^{\mathrm{A}}}{\exp \varepsilon^{\mathrm{A}}}
$$

where $F^{\mathrm{A}}$ and $\varepsilon^{\mathrm{A}}$ are the load and the true strain in region A of the sample, and $A_{0}^{\mathrm{A}}$ and $A^{\mathrm{A}}$ are the initial and current cross-sectional areas in the same region, which have installed the true stresses $\sigma^{\mathrm{A}}\left(=\sigma_{b e}+\sigma \varepsilon^{\mathrm{A}}\right)$.

Just before plastic deformation takes place in region B $\left(\varepsilon^{\mathrm{B}}=0\right.$ and $\left.\sigma^{\mathrm{B}}=\sigma_{b e}\right)$, the applied force $F$ can also be described by

$$
F=F^{\mathrm{B}}=\sigma_{b e} A_{0}^{\mathrm{B}}
$$

Taking $\Delta \varepsilon$ as the strain value that must occur in region $\mathrm{A}$ at this moment $\left(\Delta \varepsilon=\varepsilon^{\mathrm{A}}\right.$, when $\left.\sigma^{\mathrm{B}}=\sigma_{b e}\right)$, the equality of Eqs. [2] and [3] allows us to write

$$
\Delta \varepsilon=\frac{\frac{\sigma_{b e}}{\theta} \frac{\Delta A_{0}}{A_{0}^{\mathrm{B}}}}{\left(\frac{A_{0}^{\mathrm{A}}}{A_{0}^{\mathrm{B}}}-\frac{\sigma_{b e}}{\theta}\right)}
$$

To deduce this equation, it was considered that $\Delta \varepsilon$ is a relatively low strain value, and so $\exp (\Delta \varepsilon) \approx(1+\Delta \varepsilon)$; also, $\Delta A_{0}=A_{0}^{\mathrm{B}}-A_{0}^{\mathrm{A}}$. To get deformation in the entire 
Table II. Experimental Values of $\sigma_{b e}$ and $\theta$ for $\varepsilon_{p}=0.04$, $0.06,0.08$, and 0.10 *

\begin{tabular}{|c|c|c|c|c|}
\hline$\varepsilon_{p}$ & $\sigma_{b e}(\mathrm{MPa})$ & $\theta(\mathrm{MPa})$ & $\Delta A_{0}\left(\mathrm{~mm}^{2}\right)$ & $\Delta \varepsilon$ \\
\hline \multirow{4}{*}{0.04} & \multirow{4}{*}{245} & \multirow{4}{*}{400} & 0.05 & 0.008 \\
\hline & & & 0.10 & 0.016 \\
\hline & & & 0.20 & 0.033 \\
\hline & & & 0.30 & 0.050 \\
\hline \multirow{4}{*}{0.06} & \multirow{4}{*}{260} & \multirow{4}{*}{380} & 0.05 & 0.011 \\
\hline & & & 0.10 & 0.022 \\
\hline & & & 0.20 & 0.045 \\
\hline & & & 0.30 & 0.070 \\
\hline \multirow{4}{*}{0.08} & \multirow{4}{*}{272} & \multirow{4}{*}{350} & 0.05 & 0.018 \\
\hline & & & 0.10 & 0.036 \\
\hline & & & 0.20 & 0.075 \\
\hline & & & 0.30 & 0.117 \\
\hline \multirow{4}{*}{0.10} & \multirow{4}{*}{280} & \multirow{4}{*}{300} & 0.05 & 0.075 \\
\hline & & & 0.10 & 0.163 \\
\hline & & & 0.20 & 0.389 \\
\hline & & & 0.30 & 0.724 \\
\hline
\end{tabular}

*The correspondent values for $\Delta \varepsilon$ from Eq. [4] are also shown for $\Delta A_{0}=0.05,0.10,0.20$, and $0.30 \mathrm{~mm}^{2}$.

samples (regions $\mathrm{A}$ and $\mathrm{B}$ ) before necking, the strain $\Delta \varepsilon$ given by Eq. [4] must be equal to or smaller than the uniform deformation up to necking $\varepsilon_{n}$ of a homogeneous prestrained sample: $\Delta \varepsilon \leq \varepsilon_{n}$. Considering that the work-hardening behavior is given by Eq. [1] and the necking occurs, in homogeneous samples, when $d \sigma / d \varepsilon=\sigma$, it is possible to obtain the following inequality relating the mechanical behavior to the dimensional fluctuations:

$$
\frac{\Delta A_{0}}{A_{0}^{\mathrm{A}}} \leq-\left[\frac{\frac{\theta}{\sigma_{b e}}}{\frac{1}{\left(\frac{\theta}{\sigma_{b e}}\right)}-2}+1\right]
$$

So, when this equation is verified, deformation takes place in both regions before necking.

Equation [4] shows that the homogeneity of deformation at the beginning of reloading in tension depends on the degree of finishing of the samples (through the values of $\Delta A_{0}$ and $A_{0}^{\mathrm{A}}$ ) and on the plastic behavior (through the values of $\theta$ and $\sigma_{b e}$ ). The degree of finishing of the samples does not explain the difference of behavior between low and high prestrain values. In fact, the observed fluctuations of area whose dimensions were measured in width and thickness do not depend on the prestrain. Besides, the dimensions were always slightly inferior to the tolerance allowed by standardization. However, when prestrain in rolling increases, the plastic behavior changes: $\sigma_{b e}$ increases and $\theta$ decreases. Consequently, it must be expected from Eq. [4] that the beginning of deformation in region B of the sample is delayed (higher $\Delta \varepsilon$ values) for increased prestrain values. In Table II are listed the values of $\Delta \varepsilon$ calculated using Eq. [4], corresponding to four prestrain values $\left(\varepsilon_{p}=0.04,0.06\right.$, 0.08 , and 0.10 ). The values of $\sigma_{b e}$ and $\theta$ are estimated from the experimental results shown in Figures 2 and 3. As mentioned previously, the initial cross-sectional area of the tensile sample is $A_{0}^{\mathrm{A}}=10 \mathrm{~mm}^{2}$. Four values of $\Delta A_{0}$ are
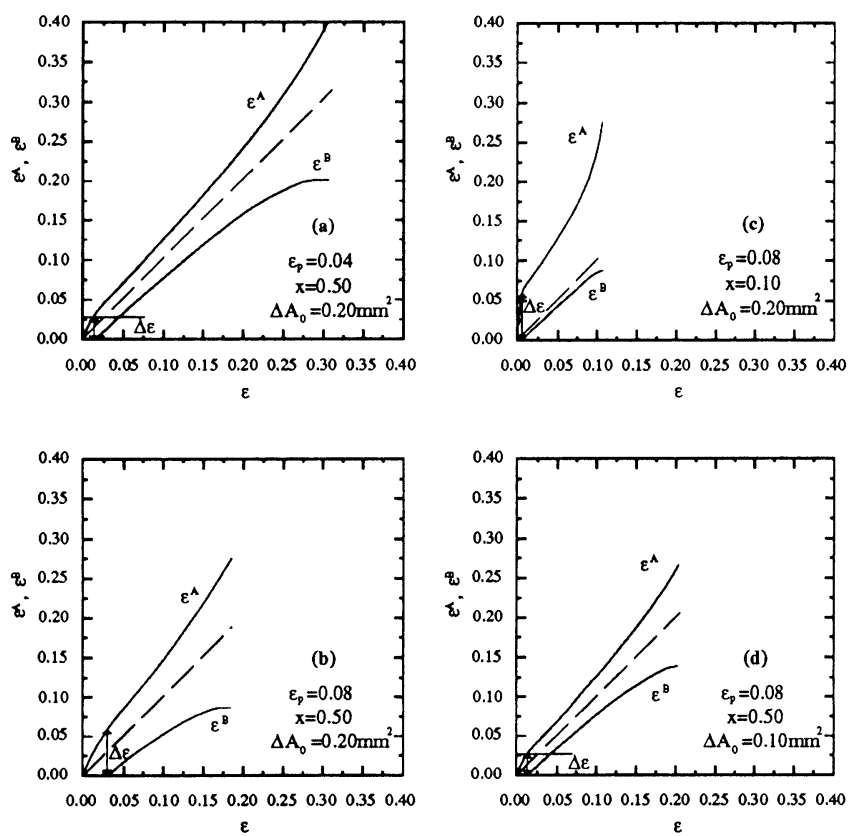

Fig. 13-Evolution of the strain in regions $\mathrm{A}\left(\varepsilon^{\mathrm{A}}\right)$ and $\mathrm{B}\left(\varepsilon^{\mathrm{B}}\right)$ with the mean strain $\varepsilon$ over the extensometer length. Four different combinations of prestrain $\left(\varepsilon_{p}\right)$ and geometrical defect $\left(\Delta A_{0}\right.$ and $\left.x\right)$ are described in the figures $(a)$ through $(d)$.

considered, which are lower than the maximum area fluctuation allowed by standardization $\left(\Delta A_{0}=0.05,0.10,0.20\right.$, and $0.30 \mathrm{~mm}^{2}$ ). For $\varepsilon_{p}=0.04,0.06$, and 0.08 , the calculated $\Delta \varepsilon$ values for $\Delta A_{0}=0.30 \mathrm{~mm}^{2}$ are close to the macroscopically observed values, for deformation to spread over the sample (as exemplified in Figure 4). The calculated results agree well with the experimental ones for this $\Delta A_{0}$ value. For the case $\varepsilon_{p}=0.10$, the deformed region enlarges at the beginning of the test, but it is not seen to attain all the sample, as predicted by Eq. [5]. So, an important partial conclusion is that the effect of fluctuations on the crosssectional area becomes significant for materials with low work-hardening and high reloading stress.

Using the previous model, it is possible to study how the nonuniform deformation observed at the beginning of the test evolves with further deformation until necking. When the strain propagates to region $\mathrm{B}$, the applied force $F$ can be calculated from Eq. [2] and from one equivalent equation concerning region $\mathrm{B}$ :

$$
F=F^{\mathrm{B}}=\left(\sigma_{b e}+\theta \varepsilon^{\mathrm{B}}\right) A^{\mathrm{B}}=\left(\sigma_{b e}+\theta \varepsilon^{\mathrm{B}}\right) \frac{A_{0}^{\mathrm{B}}}{\exp \varepsilon^{\mathrm{B}}}
$$

Using Eqs. [2] and [6], it is possible to determine successive couples $\left(\varepsilon^{\mathrm{A}}, \varepsilon^{\mathrm{B}}\right)$ for which $F=F^{\mathrm{A}}=F^{\mathrm{B}}$. Besides, the mean strain value $\varepsilon$ measured over the extensometer length (50 $\mathrm{mm}$ in the present case) can be related with $\mathcal{E}^{\mathrm{A}}$ and $\varepsilon^{\mathrm{B}}$ as follows $\left(l_{0}^{\mathrm{A}}=x l_{0}\right.$ and $l_{0}^{\mathrm{B}}=(1-x) l_{0}$, as seen in Figure 12):

$$
\begin{aligned}
\varepsilon=\ln \left(\frac{l}{l_{0}}\right)=\ln \left(\frac{l^{\mathrm{A}}+l^{\mathrm{B}}}{l_{0}}\right)= & \ln \left[x \exp \left(\varepsilon^{\mathrm{A}}\right)\right. \\
& \left.+(1-x) \exp \left(\varepsilon^{\mathrm{B}}\right)\right]
\end{aligned}
$$

Now, it is possible to plot $\mathcal{E}^{\mathrm{A}}$ and $\varepsilon^{\mathrm{B}}$ as a function of the 
mean strain $\varepsilon$ for different prestrain conditions (defined by $\sigma_{b e}$ and $\theta$ ) and geometrical defects (characterized by $\Delta A_{0}$, $A_{0}^{\mathrm{A}}$, and $x$ ), as shown in Figure 13. From the analysis of Figures 13(a) through (c), it can be concluded that for a variation in area of $0.20 \mathrm{~mm}^{2}$ (less than the mean measured value), the strain distribution in the sample is strongly dependent on prestrain values, as follows: for $\varepsilon_{p}=0.04$, when the deformation begins in region $\mathrm{B}$, the region $\mathrm{A}$ attains strain values close to 0.03 , and for $\varepsilon_{p}=0.08$, the respective value is close to 0.06 ; the difference between $\varepsilon^{\mathrm{A}}$ and $\varepsilon^{\mathrm{B}}$ clearly increases during the test for both cases. The influence of the length of the defect (characterized by $x$ ) can be understood by the comparison of Figure 13(b) and (c). As expected, when the length of the defect is small $(x=0.10)$, the measured mean strain value $\varepsilon$ represents quite well the deformation in almost the entire sample (region B). When decreasing $\Delta A_{0}$ to $0.10 \mathrm{~mm}^{2}$ (Figure 13(d)), all the previously described effects are reduced. In conclusion, the present model allowed a qualitative study of the strain distribution in prestrained samples having a geometrical defect. Moreover, a remarkable feature of the present model is the fact that it allows prediction of the strain saturation occurring in region B related to necking, as seen in Figure 13, despite the simplified work-hardening behavior and sample geometry descriptions. Finally, its results, concerning the strain distribution, qualitatively agree with the experimental ones.

The necking is a mechanical consequence of instability corresponding to the maximum load (Considere point). From the present results, the necking region coincides with the region where the deformation has been seen to start (i.e., where the initial cross section is slightly smaller), as should be expected. Furthermore, the lines that limit the necking region make an angle of between 55 to $60 \mathrm{deg}$ with the tensile axis. This corresponds to the orientation that minimum disturbances propagate. ${ }^{[34]}$ In fact, the deformation compatibility between the necking region and the rest of the sample imposes the linear deformations along the boundary direction to be equal within and outside the necking region. The strain state within the necking region tends toward a plane strain state, with the direction of zero linear deformation parallel to the direction of the lines that limit the necking. Therefore, the direction of the boundary lines must be parallel to the line of zero linear deformation in tension, which makes an angle of $54.7 \mathrm{deg}$ with the tensile direction in the case of an isotropic sheet; this is close to observed values (between 55 and $60 \mathrm{deg}$, as referred to previously).

\section{CONCLUSIONS}

The present work examines the behavior of polycrystalline tensile specimens prestrained in rolling, concerning aspects such as the microstructure, the mechanical behavior, and the strain distribution during this complex strain path.

The correlation established between the microscopic and macroscopic behaviors, after the path change, enables the following conclusions to be drawn.

1. The low work-hardening rate and high reloading yield stress, observed after path change, are due to the change of slip systems.

2. When prestrain is high enough (0.06 and greater), the transient region, observed in the nominal stress-strain curves, changes the concavity upward. For critical prestrain values, these curves present two peaks which are not commonly observed in tension.

3. Nonuniform deformation clearly observed at the beginning of reloading in tension for prestrain values of 0.06 and higher is connected with plastic behavior, through the values of the work-hardening rate and the yield stress.

4. There is no apparent connection between nonhomogeneous deformation at macroscopic level and slip behavior inside the grains. In fact, no local instabilities such as microbands or shear offsets at the grain boundaries were observed.

The nonuniform deformation observed in reloading was studied using a simplified macroscopic two-zone model. It takes into account the geometry of the defect and the mechanical behavior. This study connected with experimental results allows the following main trends to be noted.

1. The effect of fluctuations on the cross-sectional area becomes important for materials with relatively low workhardening rate and high reloading stress.

2. The evolution of the strain in both zones of the sample and the strain saturation due to necking can be well predicted by the model.

3. The macroscopic results, concerning the delay of starting deformation in some regions of the sample, are explained by the model that can predict the strain value for which such effect occurs.

4. There is a necessary analytical condition to deformation spreading over the length of the sample before necking takes place.

\section{ACKNOWLEDGMENT}

The authors are indebted to JNICT and FEDER for financial support through the STRIDE Program.

\section{REFERENCES}

1. A.K. Ghosh and W.A. Backofen: Metall. Trans., 1973, vol. 4, pp. 1113-23.

2. R.H. Wagoner and J.V. Laukonis: Metall. Trans. A, 1983, vol. 14A, pp. 1487-95.

3. K. Chung and R.H. Wagoner: Metall. Trans. A, 1986, vol. 17A, pp. 1001-09.

4. M. Zandrahimi, S. Platias, D. Price, D. Barret, P.S. Bate, W.T. Roberts, and D.V. Wilson: Metall. Trans. A, 1989, vol. 20A, pp. 247182.

5. D. Juul Jensen, A.W. Thompson, and N. Hansen: Metall. Trans. A, 1989, vol. 20A, pp. 2803-10.

6. D. Juul Jensen and N. Hansen: Acta Metall., 1990, vol. 38, pp. 136980 .

7. Y. Nakada and A.S. Keh: Acta Metall., 1966, vol. 14, pp. 961-73.

8. P.J. Jackson and Z.S. Basinski: Can. J. Phys., 1967, vol. 45, pp. 70735.

9. P. Franciosi, M. Berveiller, and A. Zaoui: Acta Metall., 1980, vol. 28, pp. 273-83.

10. U.F. Kocks, T. Hasegawa, and R.O. Scatergood: Scripta Metall, 1980, vol. 14, pp. 449-54.

11. T. Hasegawa, T. Yakou, and S. Karashima: Mater. Sci. Eng., 1975, vol. 20, pp. 267-76.

12. T. Hasegawa, T. Yakou, and U.F. Kocks: Mater. Sci. Eng., 1986, vol. 75, pp. 189-99.

13. N. Christodoulou, O.T. Woo, and S.R. MacEwen: Acta Metall., 1986, vol. 34, pp. 1553-62. 
14. M.F. Vieira, J.-H. Schmitt, J.J. Gracio, and J.V. Fernandes: J. Mater. Process. Technol., 1990, vol. 24, pp. 313-22.

15. J.-H. Schmitt, J.V. Fernandes, J.J. Gracio, and M.F. Vieira: Mater. Sci. Eng. A, 1991, vol. 147, pp. 143-54.

16. J.-H. Schmitt, E. Aernoudt, and B. Baudelet: Mater. Sci. Eng., 1985, vol. 75 , pp. 13-20.

17. J.L. Raphanel, J.-H. Schmitt, and B. Baudelet: Int. J. Plast., 1986, vol. 2, pp. 371-78.

18. J.V. Fernandes, J.J. Gracio, and J.-H. Schmitt: in Large Plastic Deformations: Fundamental Aspects and Applications to Metal Forming, C. Teodosiu, J.L. Raphanel, and F. Sidoroff, eds., A.A. Balkema, Rotterdam, 1993, pp. 219-28.

19. A.M. Dias: Coimbra University, Coimbra, Portugal, private communication, 1992.

20. J.J. Gracio, J.V. Fernandes, and J.-H. Schmitt: Mater. Sci. Eng. A, 1989, vol. 118, pp. 97-105.

21. J.V. Fernandes, J.J. Gracio, J.-H. Schmitt, and E.F. Rauch: Scripta Metall. Mater., 1993, vol. 28, pp. 1335-40.

22. J.V. Sharp and M.J. Makin: Can. J. Phys., 1967, vol. 45, pp. 519-22.

23. E.F. Rauch and J.-H. Schmitt: Mater. Sci. Eng. A, 1989, vol. 113, pp. 441-48.
24. B.V.N. Rao and J.V. Laukonis: Mater. Sci. Eng., 1983, vol. 60, pp. 125-35.

25. A. Korbel and P. Martin: Acta Metall., 1988, vol. 36, pp. 2575-86.

26. J.V. Fernandes and J.-H. Schmitt: Phil. Mag. A, 1983, vol. 48, pp. 841-70.

27. N. Hansen: Metall. Trans. A, 1985, vol. 16A, pp. 2167-90.

28. T. Leffers: in Deformation of Polycrystals: Mechanisms and Microstructures, N. Hansen, A. Horsewell, T. Leffers, and H. Lilhold, eds., Riso National Laboratory, Roskilde, Denmark, 1981, pp. 55-71.

29. U.F. Kocks and G.R. Canova: in Deformation of Polycrystals: Mechanisms and Microstructures, N. Hansen, A. Horsewell, T. Leffers, and H. Lilhold, eds., Riso National Laboratory, Roskilde, Denmark, 1981, pp. 35-44.

30. A.S. Malin and M. Hatherly: Met. Sci., 1979, vol. 137, pp. 463-72.

31. M. Hatherly and A.S. Malin: Scripta Metall., 1984, vol. 18, pp. 449-54.

32. J. Washburn and G. Murty: Can. J. Phys., 1967, vol. 45, pp. 523-39.

33. E.F. Rauch and C. G'Sell: Mater. Sci. Eng. A, 1989, vol. 111, pp. 71-80.

34. R. Hill: The Mathematical Theory of Plasticity, Oxford University Press, London, 1950, ch. XII. 